\title{
Distribution of electrophysiological abnormality in Lambert-Eaton myasthenic syndrome
}

\author{
Paul Maddison, John Newsom-Davis, Kerry R Mills
}

\begin{abstract}
Objective-To assess the distribution of electrophysiological abnormality in Lambert-Eaton myasthenic syndrome (LEMS) to identify the most sensitive muscle to use in routine examination. Methods-Surface recorded compound muscle action potential (CMAP) amplitudes were made from abductor digiti minimi, abductor pollicis brevis, anconeus, biceps brachii, and trapezius in 10 patients with LEMS. The effect of 3,4-diaminopyridine $(3,4-D A P)$ was recorded in each muscle in nine patients. CMAP amplitudes were measured at rest and immediately after 10 seconds maximal voluntary contraction in each muscle. Values were compared with results obtained from 12 healthy controls.
\end{abstract}

Results-Resting CMAP amplitudes were reduced in at least one muscle in all patients compared with controls, most markedly in abductor digiti minimi and anconeus. The administration of 3,4-DAP resulted in significantly improved resting CMAP amplitudes in trapezius only. After maximal voluntary muscle contraction, characteristic increments in CMAP amplitude of over $100 \%$ above resting values were seen in abductor digiti minimi and abductor pollicis brevis in seven patients, anconeus and biceps brachii in five patients. No patient had this level of increment in trapezius.

Conclusion-Despite predominantly proximal limb weakness seen clinically in patients with LEMS, the most sensitive muscles for detecting characteristic electrophysiological abnormalities of low resting CMAP amplitude and increment of over $100 \%$ after 10 seconds maximal voluntary contraction are abductor digiti minimi, abductor pollicis brevis, and anconeus.

(F Neurol Neurosurg Psychiatry 1998;65:213-217)

Keywords: Lambert-Eaton myasthenic syndrome; compound muscle action potential

Lambert-Eaton myasthenic syndrome (LEMS) is an autoimmune disorder of neuromuscular transmission, characterised by proximal muscle weakness, absent tendon reflexes, and autonomic dysfunction. ${ }^{12}$ About $60 \%$ of patients have an associated small cell lung cancer. ${ }^{3}$ Diagnosis of LEMS is made principally on: (1) the specific clinical features of proximal lower limb weakness, depressed tendon reflexes with posttetanic potentiation, and autonomic distur- bances, most notably dryness of the mouth ${ }^{3} ;(2)$ the presence of autoantibodies directed against P-/Q-type voltage gated calcium channels, detectable in $85 \%$ to $95 \%$ of cases $^{45}$; and (3) characteristic abnormalities found on neurophysiological examination. ${ }^{6}$

The classic diagnostic electrophysiological abnormalities in LEMS are a reduced amplitude of the resting compound muscle action potential (CMAP), a decrement in CMAP amplitude at low rates of repetitive nerve stimulation, and an increment in CMAP amplitude, typically over $100 \%$, after either high rates of nerve stimulation or 10 to $15 \mathrm{sec}-$ onds maximal voluntary contraction. ${ }^{6}$ Usually, the abductor digiti minimi muscle is used in electrophysiology to diagnose and assess the severity of LEMS, ${ }^{367}$ although several small series have recorded from trapezius, ${ }^{8}$ flexor carpi ulnaris, ${ }^{910}$ anconeus, ${ }^{11}$ tibialis anterior ${ }^{10}$ and extensor digitorum brevis. ${ }^{810}$ As the condition of LEMS is characterised by weakness that is typically proximal in nature, we aimed to define the electrophysiological range in LEMS by comparing proximal and distal recordings from five separate muscle groups in the upper limb. We used electrophysiological recordings to see if proximal muscles show a more marked abnormality on testing than distal muscles, as is often seen clinically.

\section{Materials and methods}

PATIENTS

Ten patients with LEMS were studied (five men, five women; mean age 57.7 ; range 31 to 76), all of whom were under regular follow up in our department. One patient had had a pneumonectomy for small cell lung cancer in 1989 with no subsequent recurrence of the tumour. None of the other patients had detectable malignancy (mean duration of follow up 7.8 years; range 1 to 16 years). Proximal upper limb weakness occurred during the course of the illness in all cases. At the time of initial diagnosis, all patients had the characteristic electrophysiological abnormalities of LEMS of a low resting CMAP amplitude (in abductor digiti minimi) and incremental responses of over $100 \%$ after 10 seconds maximal voluntary contraction. Antibodies to P-/Q-type voltage gated calcium channels were detected in serum samples of nine out of the 10 patients. Nine patients were treated with 3,4-diaminopyridine (3,4-DAP) at the time of testing; for these patients, initial electrophysiological recordings were made at least 10 hours after the last dose of 3,4-DAP to allow for adequate drug washout. ${ }^{12}$ In addition, eight of the patients were taking prednisolone and azathioprine and 
Table 1 Neurophysiological measurements from the right arm in patients with LEMS and controls

\begin{tabular}{|c|c|c|c|c|c|}
\hline \multirow[b]{2}{*}{ Subject } & \multicolumn{5}{|c|}{ Resting CMAP amplitudes $(m V)$} \\
\hline & $A D M$ & $A P B$ & Anconeus & Biceps & Trapezius \\
\hline \multicolumn{6}{|l|}{ Patients: } \\
\hline 1 & 3.64 & 5.6 & 0.67 & 1.24 & 4.3 \\
\hline 2 & 3.28 & 6.5 & 0.68 & 3.14 & 3.5 \\
\hline 3 & 8.6 & 17.1 & 0.86 & 5.36 & 8.6 \\
\hline 4 & 4.2 & 2.6 & 0.22 & 1.98 & 2.2 \\
\hline 5 & 1.37 & 2.64 & 0.32 & 3.54 & 4.1 \\
\hline 6 & 2.12 & 2.84 & absent & 0.29 & 4.36 \\
\hline 7 & 10.2 & 12.4 & 1.42 & 14 & 10 \\
\hline 8 & 14.6 & 2.6 & 3.56 & 0.49 & 12.3 \\
\hline 9 & 0.7 & 3.8 & 0.71 & 1.04 & 3.6 \\
\hline 10 & 4.64 & 4.08 & 0.44 & 2.8 & 9.1 \\
\hline Mean (SD) & $5.34(4.43)^{\star \star \star}$ & $6.02(4.92)^{\star}$ & $0.99(1.03)^{\star \star \star}$ & $3.39(4.04)^{\star \star}$ & $6.21(3.45)^{\star \star \star}$ \\
\hline \multirow[t]{2}{*}{ Controls } & $17.43(4.41)$ & $17.36(6.24)$ & $7.02(4.47)$ & $9.58(6.6)$ & $14.72(4.42)$ \\
\hline & (10.6 to 23.5$)$ & (4.5 to 26.6$)$ & $(1.8$ to 15.6$)$ & (1.97 to 24.6$)$ & $(7.1$ to 21.8$)$ \\
\hline
\end{tabular}

Control values are mean (SD) (range) $(\mathrm{n}=12)$.

${ }^{\star} \mathrm{p}<0.001 ;{ }^{\star \star} \mathrm{p}=0.003 ;{ }^{\star \star \star} \mathrm{p}<0.0001$; significantly reduced $v$ controls (Mann-Whitney $U$ test).

$\mathrm{ADM}=$ abductor digiti minimi; $\mathrm{APB}=$ abductor pollicis brevis.

two were taking prednisolone alone; no changes were made to these treatments before or during the study.

Identical electrophysiological studies were performed in 12 healthy controls (eight men, four women; mean age 45.4 years; range 26 to 76 years). The study was approved by the central Oxford research ethics committee.

ELECTROPHYSIOLOGICAL STUDIES

Measurements of peak to peak CMAP amplitudes were made from abductor digiti minimi, abductor pollicis brevis, anconeus, biceps brachii, and trapezius in each patient. Supramaximal electrical stimuli (pulse duration $0.1 \mathrm{~ms}$ ) were delivered using standard bipolar surface electrodes: the ulnar nerve supplying abductor digiti minimi and the median nerve supplying abductor pollicis brevis were stimulated at the wrist; the radial nerve innervating anconeus was stimulated just proximal to the elbow joint; the upper half of the brachial plexus (C5, C6, and C7) supplying biceps brachii via the musculocutaneous nerve was stimulated in the supraclavicular fossa at Erb's point, and the spinal accessory nerve innervating the trapezius was stimulated in the posterior triangle of the neck, at the midpoint of the sternocleidomastoid muscle. Circular $\mathrm{Ag} / \mathrm{AgCl}$ surface recording electrodes $1 \mathrm{~cm}$ in diameter were taped in a belly-tendon arrangement over each muscle in turn. Muscle signals were amplified (Medelec Mystro) and filtered with a bandpass of $3 \mathrm{~Hz}$ to $5 \mathrm{kHz}$. Skin surface temperature was measured with a digital thermometer (Jenway) and maintained above $32^{\circ} \mathrm{C}$ throughout the experiment. The percentage increment seen after voluntary contraction was calculated from the formula:

(CMAP (postexercise) - CMAP (preexercise)/CMAP (pre-exercise) $) \times 100 \%$

The mean result of two successive recordings was calculated for each muscle in all patients. An identical series of electrophysiological recordings were made one hour after the ingestion of $10 \mathrm{mg} 3,4-\mathrm{DAP}$ in the nine patients who were already receiving this treatment before the study.
DATA ANALYSIS

The values of resting CMAP amplitudes in each muscle and the percentage change in amplitude after 10 seconds maximal voluntary contraction in patients with LEMS were compared with normal values seen in the control patients. Statistical significance was determined using the Mann-Whitney $U$ test. The significance of the improvement in muscle responses after 3,4-DAP was also determined using Wilcoxon signed rank tests.

\section{Results}

RESTING MUSCLE RESPONSES

The amplitudes of resting muscle responses were significantly lower in all five muscle groups compared with controls, most markedly in abductor digiti minimi and anconeus (table 1). All patients with LEMS had at least one CMAP amplitude measurement that was lower than the minimum recorded response in the same muscle in controls. The distribution of electrophysiological abnormality was the same in cancer associated LEMS (patient 2; see table 1) as in patients with LEMS without cancer. Values of CMAP amplitude from proximal muscles were more variable both among patients with LEMS and controls. Mean resting CMAP amplitudes were increased in all five muscles after the administration of 3,4DAP (table 2), although this increase was only significant in the trapezius $(\mathrm{p}=0.008$, Wilcoxon signed rank test).

\section{INCREMENTAL RESPONSES}

After 10 seconds maximal voluntary contraction, increments of over $100 \%$ in CMAP

Table 2 Compound muscle action potential amplitudes measured before and one hour after 3,4-diaminopyridine

\begin{tabular}{lll}
\hline Muscle & Pre 3,4-DAP & Post 3,4-DAP \\
\hline ADM & $5.34(4.43)$ & $5.67(5.21)$ \\
APB & $6.02(4.92)$ & $8.47(8.14)$ \\
Anconeus & $0.99(1.03)$ & $1.32(1.26)$ \\
Biceps & $3.39(4.04)$ & $3.48(4.76)$ \\
Trapezius & $6.21(3.45)$ & $7.71(4.29)^{\star}$
\end{tabular}

Values are mean $(\mathrm{SD})(\mathrm{n}=9)$.

${ }^{\star} \mathrm{p}=0.008$; significantly improved following $10 \mathrm{mg} 3,4-$ diamimopyridine (Wilcoxon signed rank test).

$\mathrm{ADM}=$ abductor digiti minimi; $\mathrm{APB}=$ abductor pollicis brevis; 3,4-DAP = 3,4-diaminopyridine. 


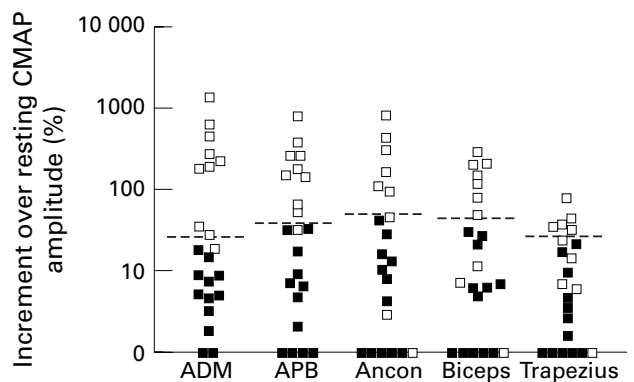

Semilogarithmic plot of increment in CMAP amplitude after 10 s maximal voluntary contraction in five upper limb muscles in patients with LEMS( $\square$ ) and controls (-).

Dashed lines represent the mean plus 2.5 SD of increment seen in control patients. Mean increments were significantly increased in LEMS compared with controls in ADM ( $p<0.0001), A P B(p<0.0001)$, anconeus $(p=0.004)$ biceps brachii $(p=0.003)$, and trapezius $(p=0.006)$. biceps brachii $(p=0.003)$, and trapezius $(p=0.006)$.
Changes in $C M A P$ amplitude $<1 \%$ are shown on the baseline; the largest decrement seen was $-28.8 \%$ recorded from the biceps muscle in one control patient. The three values from patients with LEMS recorded as $<1 \%$ were zero in anconeus, $-18.3 \%$ in biceps, and $-5.7 \%$ in trapezius. $A D M=$ abductor digiti minimi; $A P B=a b d u c t o r$ pollicis brevis; Ancon=anconeus.

amplitude above resting values were present in at least one muscle in nine of the 10 patients with LEMS. The remaining patient had increments of about $50 \%$ in abductor pollicis brevis and biceps. All control patients had increments of less than $43 \%$ in all muscle groups. The most sensitive muscle to demonstrate classic postexercise augmentation of CMAP amplitude typical of LEMS was abductor digiti minimi, with a mean increment of $348 \%$ (range $19.2 \%$ to $1414 \%$ ). The least sensitive muscle tested in the patients with LEMS was trapezius (mean increment $27 \%$; range $-5.7 \%$ to $78.2 \%$ ). Increments of $>100 \%$ were seen in seven patients in abductor digiti minimi and abductor pollicis brevis, five patients in anconeus and biceps, and in no patient in trapezius (figure). Three patients had increments $>100 \%$ in four muscles; in each case, the only muscle not to show this level of increment was trapezius. In one case (patient 7), typical post exercise CMAP amplitude increase $>100 \%$ was seen only in anconeus and a further case (patient 8) had a diagnostic increment only in abductor pollicis brevis. After 3,4-DAP, CMAP amplitude increments after maximal voluntary contraction in all five muscle groups were not significantly different than before treatment.

\section{Discussion}

In this series of patients with LEMS, we have shown that the characteristic EMG abnormalities of low resting CMAP amplitude with augmentation $>100 \%$ after maximal voluntary contraction are most commonly found in distal rather than proximal muscles. This does not correlate with the typical clinical features of proximal limb weakness, which is usually more profound than distal weakness. In a series of 50 patients with LEMS, 39 (78\%) had upper limb weakness in the course of their illness; this was irrespective of whether or not there was an associated malignancy. ${ }^{3}$ It was also noted in these patients that upper limb weakness affected proximal muscles in particular and only a few patients noticed distal weakness.

Although the major clinical feature in LEMS is proximal lower limb weakness (present in $100 \%$ of patients ${ }^{3}$ ), we did not record peripheral electrophysiological responses from proximal leg muscles for two main reasons: firstly, it is technically difficult to stimulate nerves lying deep below fascial planes proximally in the lower limb that will reproducibly evoke supramaximal muscle responses; secondly, to excite deeply situated nerves the stimulus must be of long duration and this makes the procedure unacceptably painful for the patient.

It is common practice to use abductor digiti minimi or abductor pollicis brevis for measurements of response to treatment ${ }^{12-15}$ and as a marker of severity and progression of disease. ${ }^{3}{ }^{13}$ By our comparisons with other, more proximal muscles in individual patients with LEMS, we have been able to show that abductor digiti minimi is the most sensitive muscle for detecting electrophysiological changes of LEMS and is therefore the most suitable muscle to test in studies involving patients with LEMS. Treatment with 3,4-DAP has previously been shown to improve resting CMAP amplitude in abductor digiti minimi, abductor pollicis brevis and biceps. ${ }^{12} 1415$ Our study indicates that 3,4-DAP also has a beneficial effect on anconeus and trapezius but of all five upper limb muscles tested, the only significant improvement was demonstrated in trapezius.

It is feasible that the results obtained in our experiment in patients with LEMS receiving immunosuppression are not entirely comparable with electrophysiological measurements recorded in untreated patients with LEMS. It is possible that prednisolone, in improving neuromuscular transmission, ${ }^{813}$ has a more pronounced effect on proximal rather than distal muscles. This would result in larger resting CMAP amplitudes and less marked incremental responses in proximal muscles. However, electrophysiological measurements recorded in two patients before and after starting treatment with prednisolone showed more marked improvement in resting CMAP amplitude and reduced increment in abductor digiti minimi compared to trapezius, suggesting that prednisolone does not exclusively improve the characteristic EMG abnormalities seen in LEMS in proximal muscles alone. ${ }^{8}$ In all our patients, improvement in strength was detectable clinically in both proximal and distal muscles after initiation of immunosuppression, which also resulted in improvement in bulbar and autonomic symptoms in some cases.

Several earlier studies on patients with LEMS have reported varying degrees of characteristically abnormal electrophysiological responses in different muscle groups, but these experiments were performed on only two patients. ${ }^{810}$ In another report, anconeus was compared with abductor digiti minimi and found to show more pronounced characteristic 
changes but this comparison was made in only four patients with LEMS. ${ }^{11}$

The detectable electrophysiological abnormality which is more marked distally in LEMS is by contrast with myasthenia gravis, in which studies have shown that repetitive nerve stimulation in proximal muscles such as trapezius or deltoid is more sensitive in detecting decrement of CMAP amplitude. ${ }^{16-19}$ It is possible that distal muscles of the upper limb have a lower safety factor for neuromuscular transmission than proximal muscles and therefore display greater incremental responses after exercise in LEMS. However, this does not explain why most patients have clinically detectable weakness in a proximal distribution.

During electrical stimulation of the larger proximal muscles, there is a degree of movement artefact that is not seen in particular in distal muscles and there is a risk that a stimulus given after a period of maximal voluntary contraction is not fully supramaximal as the stimulator may have moved from its original position while recording a supramaximal response at rest. This may have been a factor in the testing of one of our healthy controls, who recorded a decrement of $28.8 \%$ in biceps after voluntary contraction. One limiting factor relating to this is the degree of pain caused by stimulating deep seated nerves sufficiently to produce a supramaximal muscle response. Both patients with LEMS and control patients found stimulation of nerves supplying proximal muscles more painful than stimulation of nerves innervating distal muscles.

When stimulating the brachial plexus at Erb's point, the CMAP recorded by electrodes over the biceps may also contain contributions from other, nearby muscles that are activated by branches from the upper brachial plexus and therefore the CMAP amplitude of the biceps may not be a pure response only from this muscle. As a result, the degree of augmentation seen after exercise may not be as marked as that seen in hand muscles. However, it is surprising that trapezius, a muscle that is often weak in patients with LEMS, did not show significant augmentation after excercise in our series as the spinal accessory nerve is superficial at the point of stimulation and can be maximally stimulated with pulses of width 0.1 $\mathrm{ms}$, thereby minimising the stimulation of other surrounding muscles which may have reduced the degree of CMAP augmentation.

It has been shown that maximal voluntary contraction is more sensitive, less painful, and technically easier than $20 \mathrm{~Hz}$ repetitive nerve stimulation in detecting abnormal augmentation in LEMS $^{20}$ and was therefore the preferred method in our study. However, errors in recording maximum augmentation could have arisen because it is more difficult to be certain that maximal voluntary contraction has been achieved in proximal muscles compared with distal muscles. It is important to deliver the second stimulus immediately after the end of maximal voluntary contraction ${ }^{21}$ because the size of the incremental response diminishes greatly in the first few seconds. ${ }^{22}$ Any delay in evoking the postexercise augmented CMAP would therefore result in a falsely low increment.

Augmentation of CMAP amplitude after maximal voluntary contraction greater than $100 \%$ is not found in all muscles tested in all patients with LEMS as shown by this study and therefore it is important to establish the most sensitive muscle to test when diagnosing and monitoring severity of disease in LEMS. Patients with increments in CMAP amplitude greater than $100 \%$ in some muscles typically have more pronounced abnormalities in distal than proximal muscles. In our series, increments in every muscle in all control patients were less than $50 \%$, as were mean values plus $2.5 \mathrm{SD}$ (figure). This suggests that an incremental responses of $50 \%$ rather than $100 \%$ could be sufficient to diagnose LEMS with the same specificity over control patients.

We therefore recommend testing abductor digiti minimi at rest and after 10 seconds maximal voluntary contraction. If the findings are inconclusive, abductor pollicis brevis should be examined next and then anconeus. If the results from our series are equally applicable to those obtained when testing patients at first presentation before starting treatment, then a positive diagnosis of LEMS based on characteristic electrophysiological findings will be made in at least $90 \%$ of patients if abductor digiti minimi, abductor pollicis brevis, and anconeus are tested. This should provide a quick, effective, sensitive, and accurate electrophysiological technique for monitoring patients with LEMS. Further incremental tests on biceps and trapezius are probably not worthwhile unless there is a diagnostic uncertainty with respect to myasthenia gravis. Other, more complicated and time consuming investigations such as stimulated single fibre $\mathrm{EMG}^{23}$ may not be necessary to confirm the diagnosis.

1 Lambert EH, Elmqvist D. Quantal components of end-plate potentials in the myasthenic syndrome. Ann NY Acad Sci 1971;183:183-99.

2 Lang B, Newsom-Davis J, Wray D, et al. Autoimmune aetiology for myasthenic (Eaton-Lambert) syndrome. Lancet 981;ii:224-6.

3 O'Neill JH, Murray NMF, Newsom-Davis J. The LambertEaton myasthenic syndrome. A review of 50 cases. Brain 1988;111:577-96.

4 Motomura M, Johnston I, Lang B, et al. An improved diagnostic assay for Lambert-Eaton myasthenic syndrome. $\mathcal{F}$ Neurol Neurosurg Psychiatry 1995;58:85-7.

5 Lennon VA, Kryzer TJ, Griesmann GE, et al. Calciumchannel antibodies in the Lambert-Eaton syndrome and other paraneoplastic syndromes. N Engl f Med 1995;332: 1467-74.

6 Lambert EH, Rooke ED. Myasthenic state and lung cancer. In: Brain WR, Norris FH, eds. The remote effects of cancer on the nervous system. New York: Grune and Stratton, the nervous

7 Oh SJ, Kim DE, Kuruoglu R, et al. Electrophysiological and clinical correlations in the Lambert-Eaton myasthenic syndrome. Muscle Nerve 1996;19:903-6.

8 Ingram DA, Davis GR, Schwartz MS, et al. Cancerassociated myasthenic (Eaton-Lambert) syndrome: distribution of abnormality and effect of treatment. $\mathcal{F}$ Neurol Neurosurg Psychiatry 1984;47:806-12.

9 Oh SJ. Diverse electrophysiological spectrum of the Lambert-Eaton myasthenic syndrome. Muscle Nerve 1989; 12:464-9.

10 Oh SJ, Head T, Fesenmeier J, et al. Peroneal nerve repetitive nerve stimulation test: its value in diagnosis of myasthenia gravis and Lambert-Eaton myasthenic syndrome. Muscle Nerve 1995;18:867-73.

11 Kennett RP, Fawcett PRW. Repetitive nerve stimulation of anconeus in the assessment of neuromuscular transmission disorders. Electroencephalogr Clin Neurophysiol 1993;89: 170-6. 
12 Lundh $\mathrm{H}$, Nilsson O, Rosén I. Treatment of Lambert-Eaton syndrome: 3,4-diaminopyridine and pyridostigmine. Neu-

13 Newsom-Davis J, Murray NMF. Plasma exchange and immunosuppressive drug treatment in the Lambert-Eaton myasthenic syndrome. Neurology 1984;34:480-5.

14 Lundh H, Nilsson O, Rosén I, et al. Practical aspects of 3,4diaminopyridine treatment of the Lambert-Eaton myasthenic syndrome. Acta Neurol Scand 1993;88:136-40.

15 McEvoy KM, Windebank AJ, Daube JR, et al. 3,4 diaminopyridine in the treatment of Lambert-Eaton myasthenic syndrome. N Engl ₹ Med 1989;321:1567-71.

16 Howard JF, Sanders DB, Massey JM. The electrodiagnosis of myasthenia gravis and the Lambert-Eaton myasthenic syndrome. Neurol Clin 1994;12:305-30.

17 Schumm $F$, Stöhr $M$. Accessory nerve stimulation in the assessment of myasthenia gravis. Muscle Nerve 1984;7:147-
18 Stålberg E. Clinical electrophysiology in myasthenia gravis. 7 Neurol Neurosurg Psychiatry 1980;43:622-33.

19 Ozdemir C, Young RR. The results to be expected from electrical testing in the diagnosis of myasthenia gravis. Ann NY Acad Sci 1976;274:203-22.

20 Tim RW, Sanders DB. Repetitive nerve stimulation studies in the Lambert-Eaton myasthenic syndrome. Muscle Nerve 1994; 17:995-1001.

21 Sanders DB. Clinical neurophysiology of disorders of the neuromuscular junction. F Clin Neurophysiol 1993;10:16780.

22 Murray NMF, Newsom-Davis J. Treatment with oral 4 -aminopyridine in disorders of neuromuscular transmission. Neurology 1981;31:265-71.

23 Sanders DB. The effect of firing rate on neuromuscular jitter in Lambert-Eaton myasthenic syndrome. Muscle Nerve 1992;15:256-8.

\section{NEUROLOGICAL PICTURE}

\section{A "soap bubble" tumour in the brain: isolated cerebral immunocytoma}
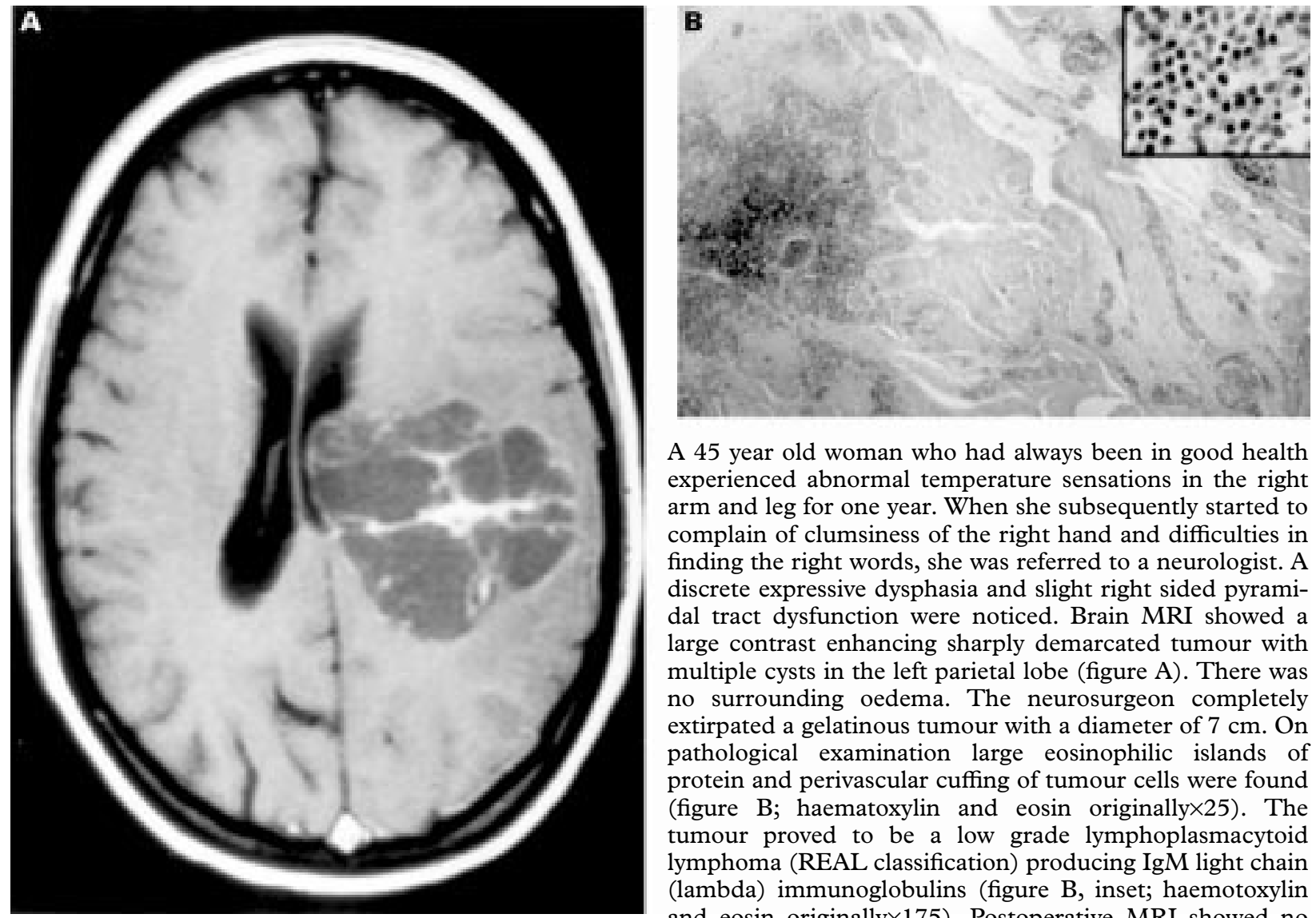

A 45 year old woman who had always been in good health experienced abnormal temperature sensations in the right arm and leg for one year. When she subsequently started to complain of clumsiness of the right hand and difficulties in finding the right words, she was referred to a neurologist. A discrete expressive dysphasia and slight right sided pyramidal tract dysfunction were noticed. Brain MRI showed a large contrast enhancing sharply demarcated tumour with multiple cysts in the left parietal lobe (figure A). There was no surrounding oedema. The neurosurgeon completely extirpated a gelatinous tumour with a diameter of $7 \mathrm{~cm}$. On pathological examination large eosinophilic islands of protein and perivascular cuffing of tumour cells were found (figure B; haematoxylin and eosin originally $\times 25$ ). The tumour proved to be a low grade lymphoplasmacytoid lymphoma (REAL classification) producing IgM light chain (lambda) immunoglobulins (figure B, inset; haemotoxylin and eosin originally $\times 175$ ). Postoperative MRI showed no residual tumour. No lymphoma was found in the bone marrow, thorax, and abdomen. Moreover, laboratory values were normal and no paraproteins were found in serum or urine. She was treated with whole brain radiotherapy.

MARTIN J B TAPHOORN CEES A F TULLEKEN GERARD H JANSEN JEAN MICHEL J KRUL 